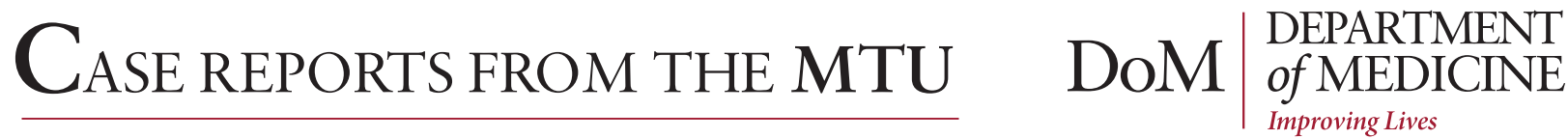

\section{Case 1. A 69 Year-Old Female Presenting With Fatigue and Acute Kidney Injury}

\author{
Nathan W.D. Lamond MD,Colin Van Zoost MD; Dennis Bowie MD \\ Department of Medicine, Dalhousie University
}

A 69 year-old female was admitted to the Medical Teaching Unit at the Queen Elizabeth II Health Sciences Centre due to acute kidney injury.

The patient had an insidious onset of weakness, anorexia, and 20-pound weight loss over four months with associated exertional dyspnea (MRC functional class II). Over the same time period, she was noted to have worsening of her previously controlled hypertension.

The patient's past medical history included impaired glucose tolerance, longstanding well controlled asthma and recently diagnosed chronic kidney disease (baseline creatinine $150 \mathrm{mmol} / \mathrm{L}$ ). Twenty years prior she had an ovarian and adrenal tumor removed, both with benign pathology. Her medications on admission included hydrochlorothiazide, ramipril, verapamil and a ventolin puffer as needed. She had no medication allergies. She was a retired teacher and did not smoke or drink alcohol.

On examination, her temperature was $36.5^{\circ} \mathrm{C}$, blood pressure $156 / 80 \mathrm{mmHg}$, heart rate 72 beats per minute, and respiratory rate 18 breaths per minute. She looked tired but was oriented to time and place. She was clinically hypovolemic with dry axilla and a jugular venous pulse below the sternal angle. She had an early-peaking systolic murmur heard best at the right second intercostal space. There was no clubbing. Equal breath sounds were heard bilaterally with no adventitious sounds. The abdomen was soft with no masses or hepatosplenomegaly. There was no palpable lymphadenopathy. The rest of her exam was unremarkable.

On admission her serum creatinine was elevated at $309 \mathrm{mmol} / \mathrm{L}$. Further investigation of this revealed elevated serum calcium $(\mathrm{sCa})$ at $3.33 \mathrm{mmol} / \mathrm{L}$ and phosphate at $1.64 \mathrm{mmol} / \mathrm{L}$, respectively. Her ionized calcium was elevated at $1.68 \mathrm{mmol} / \mathrm{L}$. The remainder of her blood work is shown in Table 1. Chest radiograph was unremarkable and ECG showed normal sinus rhythm.
She was diagnosed with acute kidney injury secondary to hypercalcemia. The ramipril and hydrochlorothiazide were discontinued as both medications may worsen acute kidney injury and hydrochlorothiazide may contribute to hypercalcemia. She was rehydrated with normal saline and following this, $90 \mathrm{mg}$ of pamidronate was administered intravenously. The $\mathrm{sCa}$ and creatinine improved quickly with this therapy.

On further investigation, parathyroid hormone was low normal and abdominal ultrasound showed normal appearing kidneys without evidence of renal calculi or hydronephrosis. However, splenomegaly with multiple hypoechogenic foci was noted incidentally. To further examine the splenic lesions, an unenhanced computed tomography (CT) scan of the chest, abdomen and pelvis was done. This revealed widespread nodules in keeping with metastatic disease involving the lungs, right hilum, liver, spleen and a single lymph node in the mediastinum. After consultation with interventional radiology none of the lesions were felt to be accessible to percutaneous biopsy. A bronchoscopy was preformed in attempt to obtain tissue diagnosis. No endobronchial lesions were identified and bronchial washings were negative for malignant cells, bacteria, fungi and acid-fast bacilli (AFB).

A presumptive diagnosis of hypercalcemia secondary to malignancy with an unknown primary was made. She was discharged from hospital in stable condition with the plan to have a positron emission tomography (PET) scan arranged as an outpatient along with thoracic surgery follow up.

Two months following her discharge she remained normocalcemic with stable renal function and therefore, required no further specific treatment. Her energy had returned back to its baseline and her blood pressure was controlled on verapamil alone. PET scan showed evidence of diffuse metastatic disease with no obvious primary (Figure 1). Based on this study it was felt that the left lobe of the liver would be amenable to percutaneous biopsy. An ultrasound-guided percutaneous biopsy was performed and revealed several large non-necrotizing granulomas (Figure 2). Special stains were negative 
for AFB and fungal organisms. Given the pathological findings a final diagnosis of sarcoidosis was made which accounted for both the hypercalcemia and widespread parenchymal nodules.

She continued to have stable exertional dyspnea with pulmonary function tests showing mild airflow obstruction. This was felt to be secondary to mild asthma and treated with inhaled corticosteroids. She had no other organ symptomatology that required specific therapy for sarcoid but continued to be followed by respirology. One year following her admission to hospital she was found to have asymptomatic mild hypercalcemia. She was encouraged to maintain adequate oral hydration and prednisone $20 \mathrm{mg}$ daily was started in an attempt to control her sarcoidosisinduced hypercalcemia.

\section{Discussion}

Hypercalcemia is a commonly encountered and heterogeneous diagnosis that may be defined as a $\mathrm{SCa}$ measurement greater than $2.5 \mathrm{mmol} / \mathrm{L}$. Due to the highly protein-bound nature of $\mathrm{sCa}$, this measurement must be corrected for serum albumin concentration or verified using an ionized calcium measurement. This allows for pseudohypercalcemia, a falsely high $\mathrm{sCa}$ measurement due to elevated albumin concentrations, to be ruled out. Clinically, the presentation of hypercalcemia depends upon its numerical severity. Mild hypercalcemia ( $\mathrm{sCa}=2.5-3.0)$ generally remains asymptomatic until discovery during routine testing or investigation of symptoms related to its underlying etiology; whereas, severe hypercalcemia ( $\mathrm{SCa}>3.5$ ) is more likely to present to medical attention and may be life-threatening. Presentations attributed to

Table 1. Laboratory Data.

\begin{tabular}{|c|c|c|c|c|c|}
\hline Variable & $\begin{array}{l}\text { Reference } \\
\text { Range }\end{array}$ & Admission & $\begin{array}{l}2 \text { Days } \\
\text { Post- } \\
\text { Admission }\end{array}$ & Discharge & $\begin{array}{l}1 \text { Year } \\
\text { Post- } \\
\text { Discharge }\end{array}$ \\
\hline Hemoglobin $\times 10^{9} / \mathrm{L}$ & $120-160$ & 115 & 104 & 86 & 118 \\
\hline White cell count $\times 10^{9} / \mathrm{L}$ & $4.5-11.0$ & 5.6 & 4.0 & 6.7 & 6.3 \\
\hline Platelet count $\times 10^{9} / \mathrm{L}$ & $150-350$ & 160 & 177 & 204 & 142 \\
\hline Sodium (mmol/L) & $136-144$ & 138 & 139 & 134 & \\
\hline Potassium (mmol/L) & $3.6-5.1$ & 3.8 & 3.6 & 4.1 & \\
\hline Chloride (mmol/L) & $101-111$ & 101 & 107 & 103 & \\
\hline Total $\mathrm{CO}_{2}(\mathrm{mmol} / \mathrm{L})$ & $22-32$ & 29 & 25 & 25 & \\
\hline Urea (mmol/L) & $2.9-9.3$ & 21.8 & 13.5 & 10.7 & 13.1 \\
\hline Creatinine (mmol/L) & $37-96$ & 309 & 243 & 148 & 130 \\
\hline lonized calcium (mmol/L) & $1.07-1.41$ & 1.68 & 1.37 & 136 & \\
\hline Calcium (mmol/L) & $2.23-2.58$ & 3.33 & 2.51 & 2.5 & 2.78 \\
\hline $\mathrm{PO}_{4}(\mathrm{mmol} / \mathrm{L})$ & $0.76-1.53$ & 1.64 & 1.2 & 1.10 & 0.92 \\
\hline PTH (pmol/L) & $1.5-10.2$ & 2.6 & & & 1.7 \\
\hline Total protein $(g / L)$ & $61-81$ & 56 & & & 72 \\
\hline Albumin $(g / L)$ & $35-50$ & 37 & 35 & 42 & 40 \\
\hline \multicolumn{6}{|l|}{ Urinalysis } \\
\hline Protein $(g / L)$ & None-trace & 0.3 & & & 0.3 \\
\hline Blood & None & None & & & None \\
\hline SPEP & & Normal & & & Normal \\
\hline
\end{tabular}

$\mathrm{CO}_{2}$ : carbon dioxide, $\mathrm{PO}_{4}$ : phosphate, $\mathrm{PTH}$ : parathyroid hormone, SPEP: serum protein electrophoresis. 
Table 2. Clinical presentations of hypercalcemia.

Renal

Nephrolithiasis

Nephrocalcinosis, chronic kidney disease

Nephrogenic diabetes insipidus

Polyuria, nocturia, prerenal acute kidney injury

\section{Gastrointestinal}

Abdominal pain, nausea

Constipation

Anorexia, weight loss

Pancreatitis

\section{Skeletal}

Bone pain

Osteoporosis

\section{Neurological}

Impaired cognition

Fatigue

Weakness

Decreased level of consciousness

\section{Cardiovascular}

Hypertension

Bradycardia

Shortened QT interval

Arrhythmia

hypercalcemia are shown in Table 2. Clinical assessment of the hypercalcemic patient begins with identification of these sequelae but must be accompanied by a search for symptoms and signs of the underlying etiology.

In practice the most common etiologies of hypercalcemia are primary hyperparathyroidism and malignancy, two diseases that present quite differently. ${ }^{1}$ Isolated hyperparathyroidism generally causes mild, asymptomatic hypercalcemia that is detected on unrelated investigations. In contrast, malignancy may present with severe, symptomatic hypercalcemia along with symptoms due to the malignancy itself. Common malignancies include: multiple myeloma,

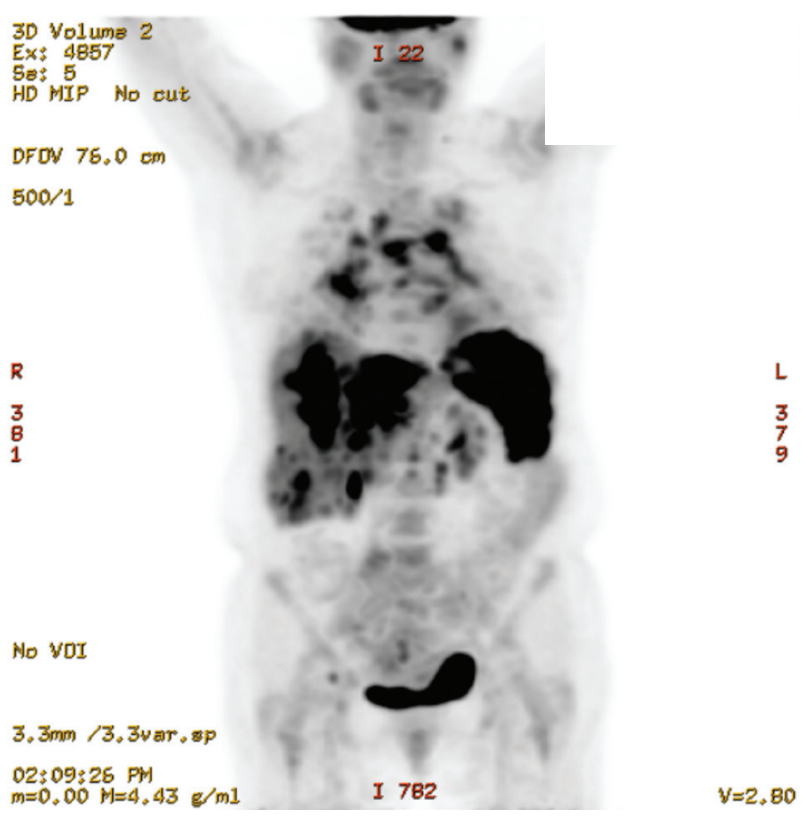

Figure 1. PET scan image showing diffuse disease involving the liver, spleen, lung and mediastinal lymph nodes. Normal physiologic uptake is noted in the bladder, kidneys, and brain.

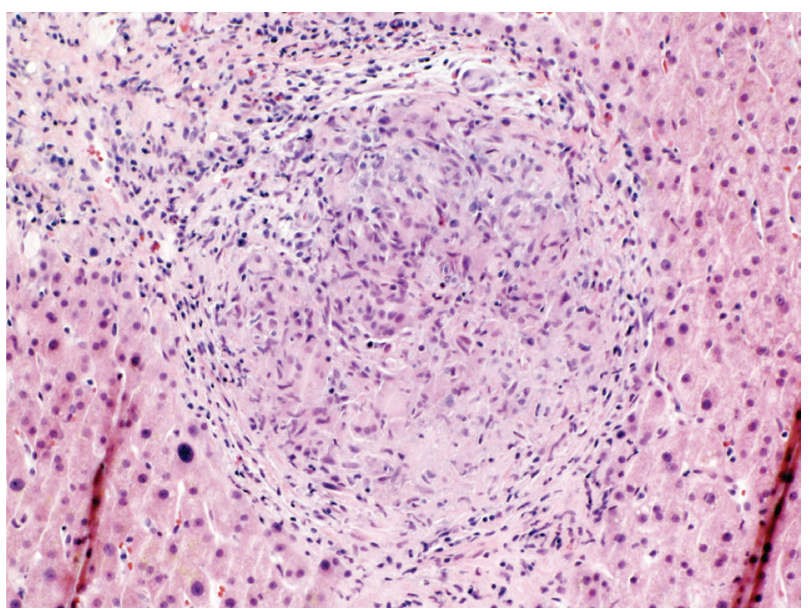

Figure 2. Liver biopsy showing a non-necrotizing granuloma with many giant cells, characteristic of sarcoidosis.

lung cancer, breast cancer, and lymphoma. While primary hyperthyroidism and malignancy account for approximately $90 \%$ of hypercalcemia, there exist numerous other causes. Recently there has also been increased recognition of multifactorial hypercalcemia, including coexistence of primary hyperparathyroidism and malignancy. ${ }^{1}$ Due to the broad differential and the potential for multifactorial etiologies, an organized approach to the underlying cause of hypercalcemia is essential. The authors' preferred approach is shown in Figure 3 and consists of a differential diagnosis guided by clinical findings and relying on measurements of 


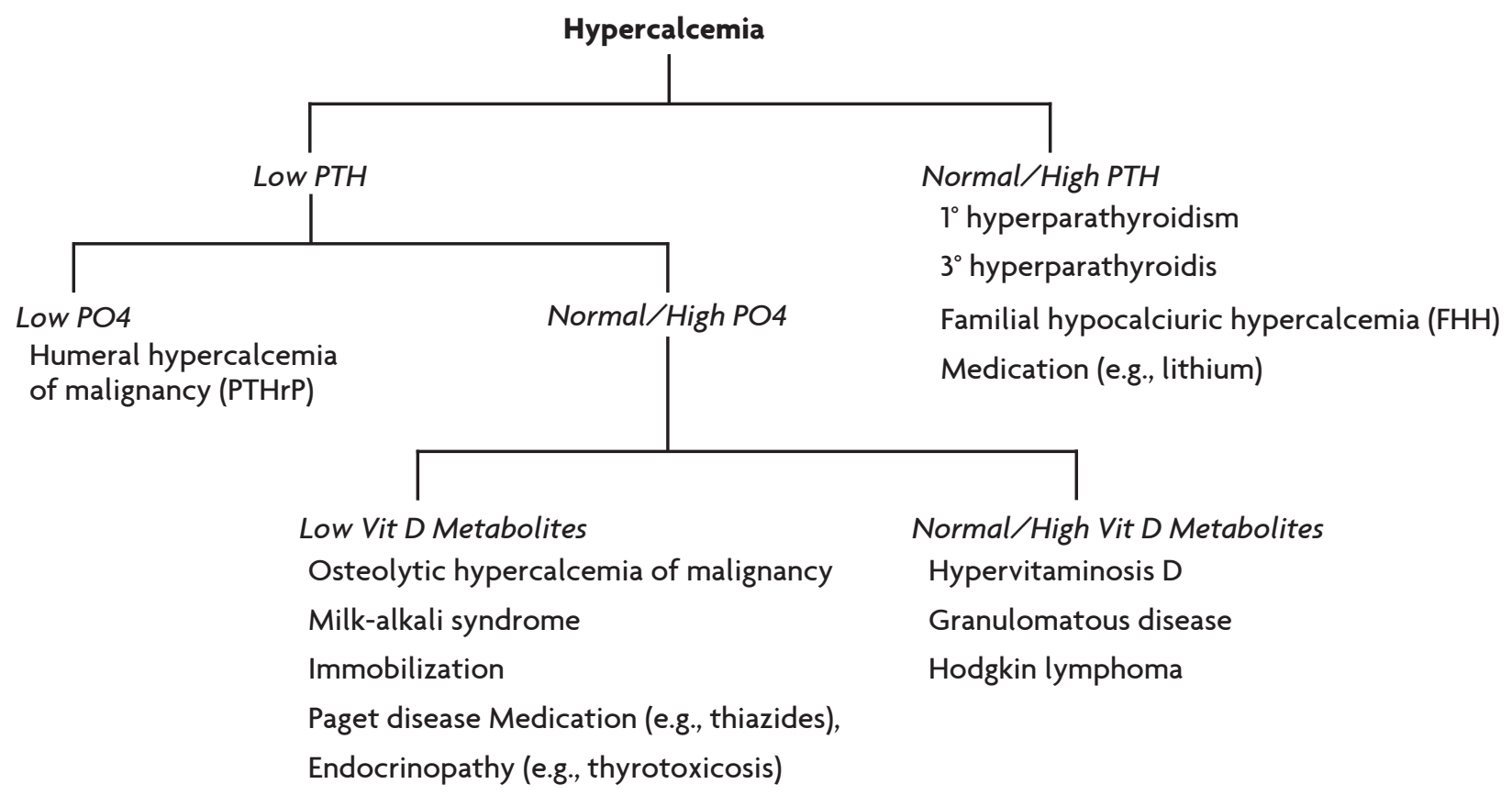

Figure 3. Diagnostic approach to the etiology of hypercalcemia. PTHrP: parathyroid hormone-related peptide, PTH: parathyroid hormone, Vit D: vitamin D

serum PTH, PO4, and Vit D metabolites. These results are then used to guide further investigations based on a narrowed differential diagnosis. The present case is a clear example of this idiom, as early diagnostic workup of acute kidney injury revealed imaging findings of disseminated parenchymal nodules, which guided further investigations. Diagnosing the underlying cause of hypercalcemia often requires days, therefore initial management of the hypercalcemia is uniformly empiric. In contrast, chronic management relies on both treatment of the underlying etiology, as well as etiology-specific treatment of hypercalcemia.

In the present case hypercalcemia was secondary to sarcoidosis, an idiopathic multisystem disease characterized by non-necrotizing granulomatous inflammation. Sarcoidosis has been observed across all races and demographics, though its incidence peaks at 20 to 39 years of age and women are more frequently affected than men. ${ }^{2}$ In North America, annual incidence depends upon race, with Blacks more commonly affected than Caucasians (35.5 vs. 10.9 cases per 100,000, respectively). ${ }^{2}$ Like hypercalcemia, sarcoidosis is associated with a wide variety of clinical presentations ranging from asymptomatic hilar adenopathy discovered on routine chest $x$-ray, to organthreatening disease. Among potential presentations, constitutional symptoms (including severe fatigue) and intra-thoracic lymph node enlargement are common. Other specific complications and patient prognosis depend upon organ system involvement with the most commonly affected organs being lungs, eyes, and skin. Prognosis is most directly determined by pulmonary disease and is generally favourable. Up to two thirds of those diagnosed with sarcoidosis undergo lasting spontaneous remissions with no identifiable disease consequences. ${ }^{3}$ As such, systemic sarcoidosis treatment is only indicated for organ-threatening disease. ${ }^{3}$ The mainstay of management is glucocorticoids, though evidence is accumulating on the use of other immunosuppressive agents in organ-specific treatment regimens. ${ }^{4}$

The reported incidence of hypercalcemia in sarcoidosis varies considerably. While several early studies reported much higher estimates, the recent Case Control Etiologic Study of Sarcoidosis (ACCESS) found that $3.7 \%$ of 736 patients had hypercalcemia. ${ }^{5}$ Many more sarcoid patients are affected by a related disorder, hypercalciuria, which may be organ-threatening due to nephrocalcinosis and nephrolithiasis. With similar pathophysiological mechanisms, hypercalciuria and hypercalcemia may be considered points along the same spectrum of disease. Like most diseases, calcium disorders in sarcoidosis seem to have a complicated underlying pathophysiology. Production of PTH-related peptide (PTHrP) in granulomata has recently been demonstrated in a subset of hypercalcemic sarcoidosis patients. ${ }^{6}$ However, conclusive evidence also exists documenting autologous, extrarenal production of $1 \alpha$-hydroxylase among activated macrophages in sarcoid granulomata. ${ }^{7}$ Autologous $1 \alpha$-hydroxylase 
production in turn leads to increased calcitriol production, elevating serum calcium levels via several effects. This $1 \alpha$-hydroxylase production is felt to be the most important mechanism leading to calcium disorders in sarcoidosis and should be addressed directly in its chronic management. As such, all patients should be instructed to limit vitamin D and calcium intake along with maintaining intravascular volume. This helps to prevent the positive feedback loop created by calciuresis, which leads to intravascular volume depletion and further increases in serum calcium levels. ${ }^{7}$ If these measures are insufficient to control hypercalcemia (or significant hypercalciuria), then pharmacologic treatment is indicated. Similar to other manifestations of sarcoid, moderate dose glucocorticoids (prednisone 10 to $40 \mathrm{mg}$ daily) are the first line treatment for hypercalcemia. Glucocorticoids have been shown to decrease $1 \alpha$-hydroxylase release from granulomata, leading to decreased serum calcium levels within 3 to 5 days. $^{7}$ Due to this rapid activity, failure to achieve normocalcemia after 2 weeks of steroid treatment should prompt consideration of concomitant disorders. However, glucocorticoid-resistant, sarcoidosis-induced hypercalcemia has been described in the literature and may respond to ketoconazole, hydroxychloroquine, and/or bisphosphonates. ${ }^{7}$

Malignancy was felt to be the likely cause of hypercalcemia in the present case given its common association with hypercalcemia and our patient's widespread parenchymal nodules. Sarcoidosis, due to its multisystem nature, can mimic many other diseases including metastatic malignancy as was observed in this case. Several similar cases (including acute kidney injury, hypercalcemia, and metastatic nodules) have been reported of patients with clinical and investigational features consistent with malignancy but ultimately were diagnosed with sarcoidosis. ${ }^{8}$ Granulomatous nodules caused by sarcoidosis can involve virtually any organ, lymph node, or bone and be indistinguishable from malignancy on traditional imaging studies. As seen in this case, sarcoidosis lesions are also fluorodeoxy-glucose avid on PET scan, similar to malignancy (along with other inflammatory and infectious disorders). ${ }^{9}$ These similarities underline the importance of:

1) considering sarcoidosis in the differential diagnosis for hypercalcemia and widespread nodules; and,

2) basing all treatment decisions on tissue diagnoses.

\section{Conclusion}

Hypercalcemia should be considered early in the differential diagnosis of acute kidney injury and if found should be approached in a systematic fashion. While sarcoidosis is a rare cause of acute kidney injury due to hypercalcemia, it is one of several granulomatous diseases that cause hypercalcemia through release of $1 \alpha$-hydroxylase. Finally sarcoidosis may also present with widespread parenchymal nodules, mimicking malignancy.

\section{Acknowledgement}

The authors would like to thank Dr. Erica Schollenberg for her help in the preparation of this manuscript.

\section{References}

1. Casez JP, Pfammatter R, Nguyen Q, Lippuner K, Jaeger P. Diagnostic approach to hypercalcemia: relevance of parathyroid hormone and parathyroid hormone-related protein measurements. Eur J Internal Med 2001;12:344-349.

2. Rybicki BA, Major M, Popovich J Jr, Maliarik MJ, Iannuzzi MC. Racial differences in sarcoidosis incidence: a 5-year study in a health maintenance organization. Am J Epidemiol 1997;145:234241.

3. Ianuzzi MC, Rybicki BA, Teirstein AS. Sarcoidosis. N Engl J Med 2007;357:2153-2165.

4. Paramothayan S, Lasserson TJ, Walters EH. Immunosuppressive and cytotoxic therapy for pulmonary sarcoidosis. Cochrane Database Syst Rev 2006;3:CD003536.

5. Baughman RP, Teirstein AS, Judson MA, et al. Clinical characteristics of patients in a case control study of sarcoidosis. Am J Respir Crit Care Med 2001;164:1885-1889.

6. Zeimer HJ, Greenaway TM, Slavin J, et al. Parathyroid-hormonerelated protein in sarcoidosis. Am J Pathol 1998;152:17-21.

7. Sharma OP. Vitamin D, calcium, and sarcoidosis. Chest 1996;109:535-539.

8. Waanders F, Hengel P, Krikke A, Wessiling J, Nieboer P. Sarcoidosis mimicking metastatic disease: a case report and review of the literature. Netherlands J Med 2006;64:342-345.

9. Lewis PJ, Salama A. Uptake of fluorine-18-fluorodeoxyglucose in sarcoidosis. J Nucl Med 1994;35:1647-1649. 